\title{
Generación de frentes de onda acústicos cilíndricos a través de parlantes iónicos
}

Cylindrical acoustic wavefront generation by means of ionic speakers

\author{
P. Gomez*, E. M. D’Onofrio*, G. D. Santiago ${ }^{\dagger 1}$ \\ *Universidad de Buenos Aires, Facultad de Ingeniería,Departamento de Electrónica \\ Paseo Colón 850, C1063ACV, Buenos Aires, Argentina \\ ${ }^{\dagger}$ Universidad de Buenos Aires, Facultad de Ingeniería, \\ Grupo de Láser, Óptica de Materiales y Aplicaciones Electromagnéticas (GLOMAE) \\ Paseo Colón 850, C1063ACV, Buenos Aires, Argentina \\ ${ }^{1}$ gsantiaefi.uba.ar \\ Recibido: 04/10/18; Aceptado: 09/11/18
}

\begin{abstract}
We present the use of ionic transducers to generate acoustic waveforms of cylindrical shape. We resorted to "cold discharge" transducers where momentum transfer from ions to neutral molecules is the main sound-generating process. Since the electrodes can be given a fairly arbitrary shape and the airflow is normal to the former, it is possible to obtain, close to the electrodes, a wavefront quite different from those obtained with standard, piston-like, loudspeakers. We present the acoustical characteristics of a cylindrical transducer and the use in the study of a bandpass, soniccrystal based filter.
\end{abstract}

Keywords: ionic transducers, acoustic radiation, sonic crystals.

Resumen- Presentamos la generación de frentes de onda acústicos cilíndricos usando transductores iónicos. Estos transductores están basados en la transferencia de momentum lineal por colisiones entre iones generados en una descarga corona y moléculas neutras. Dado que la forma de los electrodos puede ser elegida con gran flexibilidad, es posible obtener un flujo de aire que, en las vecindades del emisor, tiene asociado un frente de onda que se aparta de los conseguidos habitualmente con pistones planos. Mostramos las características acústicas de un transductor cilíndrico y, como aplicación, el uso en el estudio de un filtro pasabanda basado en un cristal sónico.

Palabras clave: transductores iónicos, radiación acústica, cristales sónicos

\section{INTRODUCCIÓN}

Los parlantes convencionales de bobina móvil se comportan, en primera aproximación, como un pistón circular plano. Si el mismo se encuentra montado en una pared infinita el diagrama de presión en el campo lejano sigue una ley de la forma [1]:

$$
p(\alpha)=C \frac{J_{1}(k \alpha)}{k \alpha}
$$

donde $\alpha$ es el ángulo respecto de la normal al pistón, $C$ y $k$ dependen de las dimensiones y la frecuencia, y $J_{1}$ es la función de Bessel de primer orden.

Por otra parte, en el campo cercano la presión resultante sigue una ley más compleja, determinada por el número de Fresnel del emisor en la posición de recepción. Conforme las dimensiones del emisor disminuyen el mismo semeja una fuente puntual. Las fuentes puntuales son simples de analizar y, en principio, con una superposición de éstas, es posible alcanzar un diagrama de emisión arbitrario. Lamentablemente, cuando un parlante se torna cuasi-puntual la resistencia de radiación disminuye drásticamente y con ello la eficiencia. Por otra parte, el número de emisores necesarios para conseguir un dado frente de onda puede tornarse excesivamente grande.

En los transductores iónicos el campo de velocidades del aire en las vecindades del emisor sigue la forma de los electrodos, por lo que es posible conformar de manera sencilla la forma del frente de onda.

En este artículo mostramos el desarrollo y caracterización de un parlante iónico cilíndrico el cual fue utilizado para medir las propiedades de un filtro acústico pasabanda basado en uncristal sónico.

\section{PRINCIPIO DE FUNCIONAMIENTO DEL PARLANTE IÓNICO}

Existen varios tipos de transductores iónicos aunque muy pocos artículos que tratan analíticamente la temática [2]-[4]. El prototipo construido consta de dos grupos de electrodos entre los cuales se aplica una alta tensión continua. La misma debe ser lo suficientemente elevada para que se produzca una descarga eléctrica entre los electrodos. Según la geometría de los electrodos y las condiciones de excitación se pueden obtener distintas descargas, siendo la del tipo corona el objeto de este estudio. En la Fig. 1 se muestra un esquema conceptual de la descarga. El electrodo de la izquierda, de diámetro pequeño, se encuentra conectado a un alto voltaje negativo respecto del electrodo de la derecha (de gran radio de curvatura). El alto campo eléctrico en el entorno del electrodo de la izquierda determina la emisión de electrones que, a su vez, generan otros electrones e iones positivos a través de colisiones. Esta descarga no entra en un proceso de avalancha porque el campo eléctrico es lo suficientemente intenso como para provocar ionizaciones sólo en una pequeña región próxima al electrodo de la izquierda. Los iones positivos derivan hacia el electrodo negativo y los electrones son capturados por moléculas neutras para formar iones negativos que derivan hacia el electrodo de la derecha. 


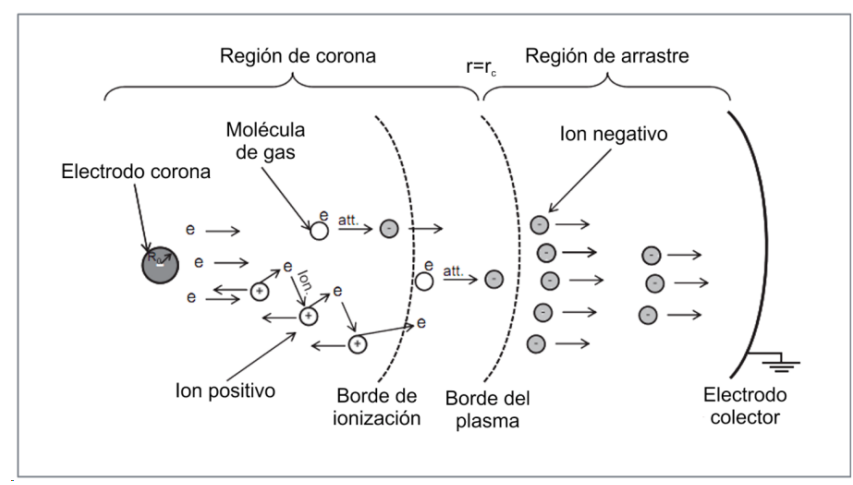

Fig. 1. Esquema conceptual de la descarga

La descarga corona mencionada en el párrafo anterior es auto-sostenida. Tiene la particularidad de que el campo eléctrico es inhomogéneo $\mathrm{y}$, como ya se mencionó, sólo genera ionización en una pequeña región limitada al entorno del denominado electrodo corona. La inhomogeneidad del campo deviene de los diferentes radios de curvatura de los electrodos. Esta descripción aplica estrictamente a un régimen de corriente continua si bien existen también descargas coronas a frecuencias industriales y de radiofrecuencia. En este trabajo nos restringimos al régimen de corrientes unipolares.

Una característica importante de la descarga corona a presión atmosférica es la aparición del denominado viento eléctrico [5]. El origen de este viento se encuentra en las colisiones entre los iones y las moléculas neutras en las cuales se transfiere momentum lineal. De esta forma, las moléculas neutras se mueven en la misma dirección que los iones generando así el transporte de aire.

$\mathrm{Si}$ se modula la tensión aplicada superponiendo una componente alterna (señal de audio) a la continua, una onda acústica es generada en el medio a través de dos mecanismos diferentes. Uno de ellos ocurre en la zona de deriva en un proceso similar al del ya mencionado viento eléctrico. Allí la señal de audio modula la velocidad de los iones que luego transfieren momentum al medio a través de colisiones. El otro mecanismo se sitúa en la zona de ionización donde la señal alterna genera cambios de temperatura y éstos se traducen en cambios de presión

Los parlantes donde predomina la transferencia de momentum se denominan de "plasma frío". Los parlantes iónicos por descarga corona están incluidos en esta categoría. En el caso de producirse un arco entre los electrodos, solamente se tiene producción de sonido por cambios de temperatura. Por lo tanto, la descarga de arco eléctrico es el principio que utilizan los llamados parlantes de "plasma caliente".

\section{CONSTRUCCIÓN}

Los parlantes iónicos, al no depender de una membrana para la generación del sonido sino en grupos de electrodos, permiten experimentar con diversas topologías para conformar el frente de onda. La primera de ellas se encuentra en el artículo publicado en 1955 por Tombs [6]. El mismo consistía en una aguja (electrodo corona) y una rejilla metálica (electrodo colector). En el mismo artículo y luego

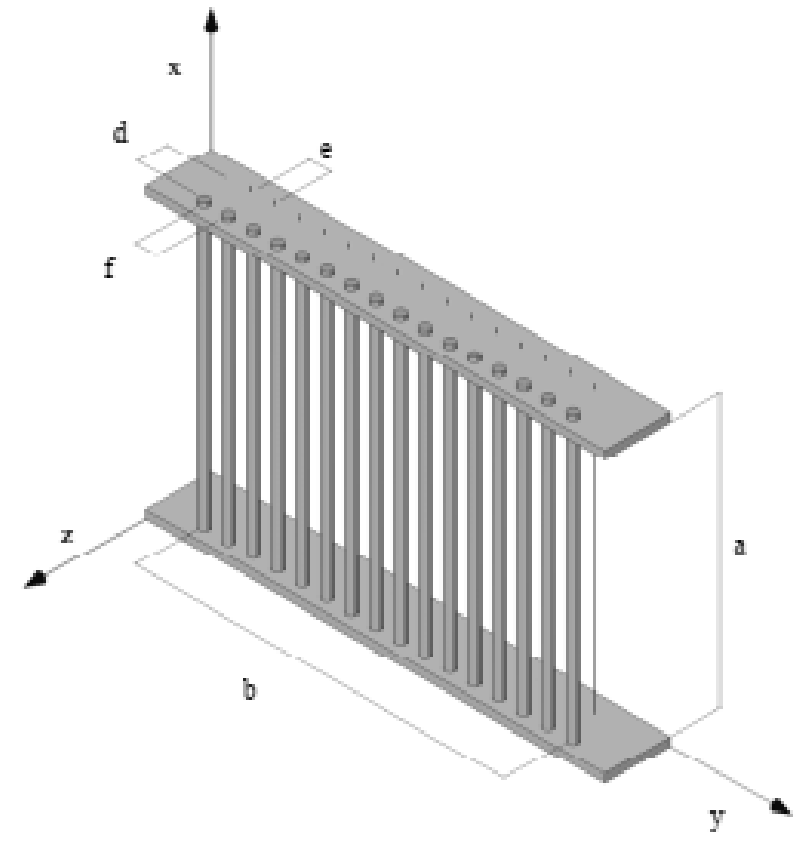

Fig. 2. Esquema del emisor plano

en posteriores diseños, este concepto se extendió a arreglos bidimensionales de puntas para lograr mayor superficie ionizada [7]. La construcción y experimentación con estos diseños permitió obtener un conocimiento preliminar muy valioso, pero para lograr niveles de presión sonora mayores decidimos ensayar otra forma de los electrodos. En lugar de utilizar puntas de aguja como electrodo corona, empleamos alambres muy delgados. Inicialmente empleamos nichrome de $50 \mu \mathrm{m}$ de diámetro pero la fragilidad e imposibilidad de soldarlos nos llevaron a cambiar a hilo de cobre de $100 \mu \mathrm{m}$ de diámetro. Como electrodo colector se utilizaron barras de bronce de $3 \mathrm{~mm}$ de diámetro, ubicadas paralelas a los hilos del electrodo corona. Curiosamente, no encontramos una caracterización detallada de un arreglo de electrodos tan simple como éste, dentro del alcance de nuestra búsqueda bibliográfica. Surge como idea directa que así se conseguirá un mayor volumen ionizado, en comparación con un arreglo de puntas discretas. Así se obtiene una mayor superficie de ionización y con ello aumenta el volumen de aire desplazado y la presión sonora. El primer prototipo construido fue un arreglo plano de electrodos en el que verificamos que el mismo irradiaba, en el campo lejano, como un pistón rectangular sólido [8]. En la Fig. 2 puede verse un esquema de CAD y en la Fig. 3 una fotografía del prototipo.

Vista la posibilidad de dar forma más compleja al arreglo, decidimos desarrollar una fuente cuyo frente de onda fuera cilíndrico. El prototipo está constituido por 16 barras de bronce de $16 \mathrm{~cm}$ de largo y $3 \mathrm{~mm}$ de diámetro (electrodos colectores), dispuestas sobre la periferia de una circunferencia de $42 \mathrm{~mm}$ de diámetro que encierran la misma cantidad de hilos de cobre (electrodos corona) a una distancia interelectródica de $11 \mathrm{~mm}$. En la Fig. 4 se pueden ver fotografías y el modelo CAD de este prototipo.

Para que el arreglo sea funcional, se deben agregar 


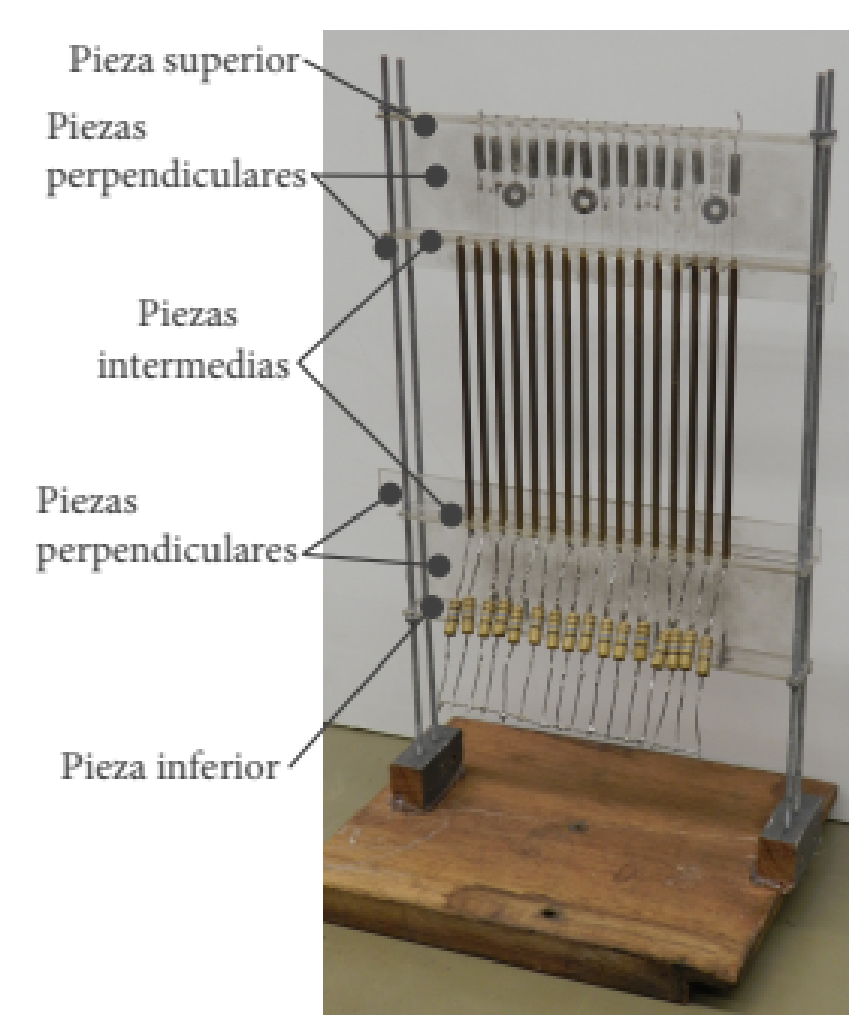

Fig. 3. Emisor plano. Nótese los resortes que tensan los electrodos corona y las resistencias ecualizadoras
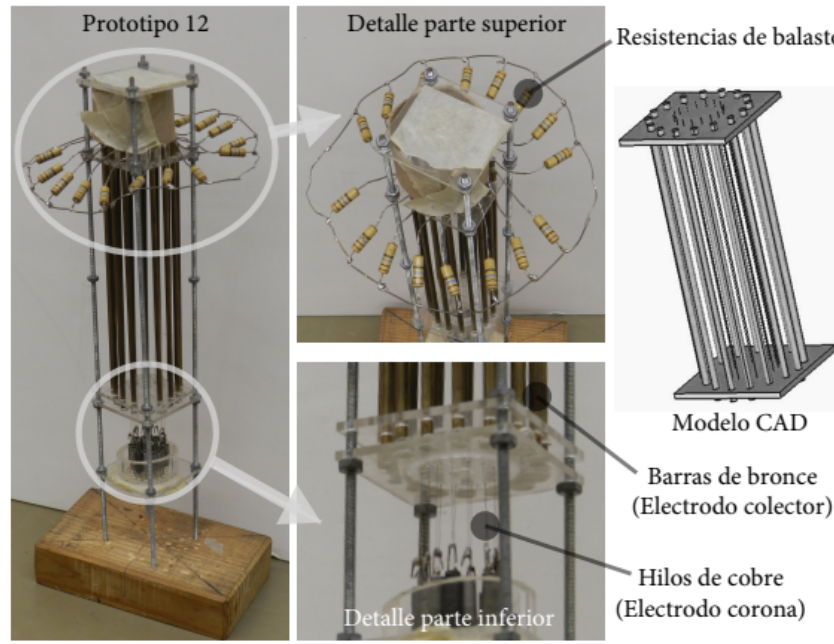

Fig. 4. Esquema conceptual y fotografía del emisor cilíndrico

algunos elementos extra. A cada una de las barras de bronce se suelda una resistencia de balasto $\left(R_{l}\right)$ para ecualizar las corrientes y también limitarlas en caso de producirse un arco eléctrico. Estas resistencias por sí solas disminuirían el nivel de señal que es aplicado sobre las barras. Para evitar esto se agregan capacitores de desacople $\left(C_{d}\right)$ en paralelo a las resistencias de balasto (no mostrados en la fotografía). De esta manera se logra inyectar la señal de audio directamente en las barras. Para dimensionar los capacitores buscamos que la reactancia de los mismos, a las frecuencias de interés, fuera despreciable frente a la resistencia.

Finalmente los electrodos corona se conectan en paralelo

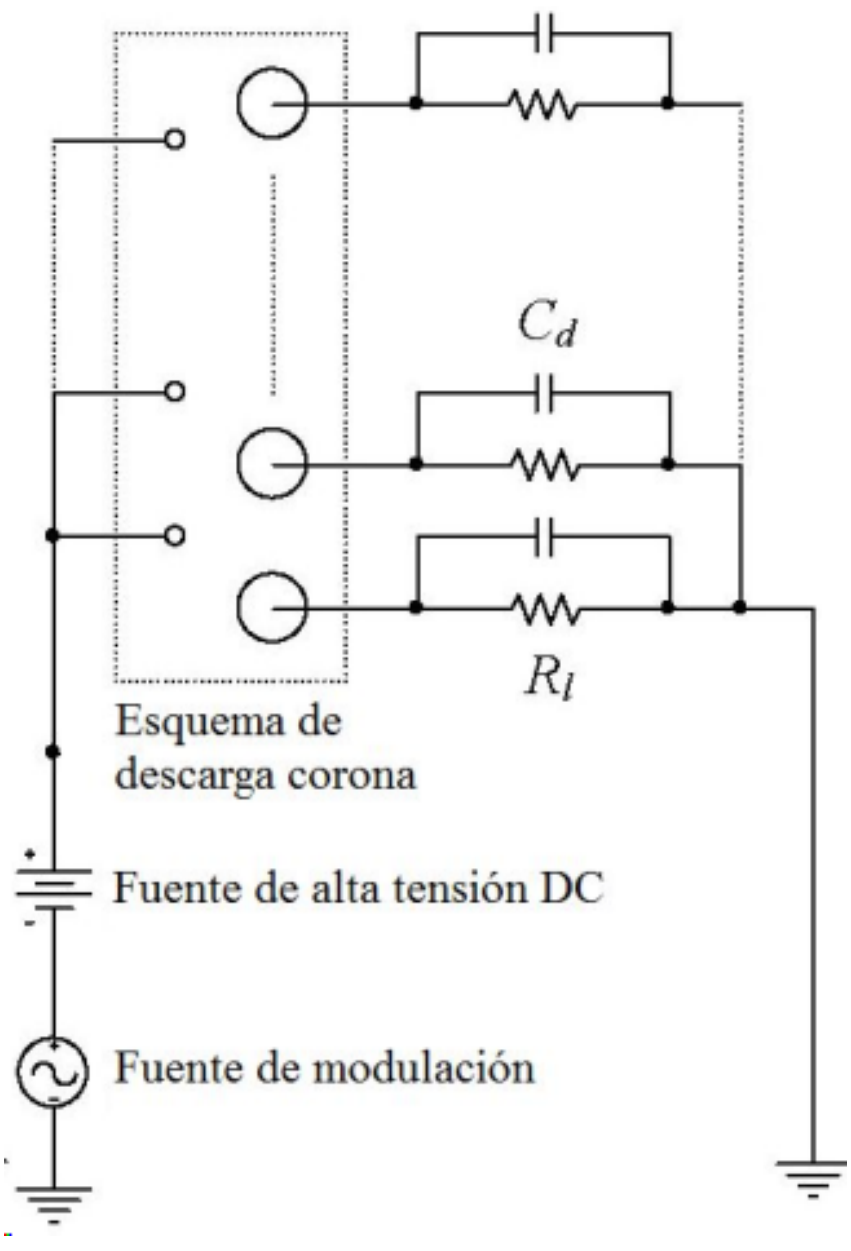

Fig. 5. Esquema del circuito eléctrico

entre sí y los terminales libres de las resistencias de balasto también se conectan en paralelo. En la Fig. 5 se observa el esquema eléctrico del dispositivo.

\section{CARACTERÍSITCA ACÚSTICA}

Para caracterizar acústicamente una fuente es necesario medir el sonido emitido libre de las reflexiones que se originan, por ejemplo, dentro de un recinto. Cuando esta condición no se tiene en cuenta, el micrófono recibe la señal directa junto con las reflejadas dando lugar a efectos de interferencia que llevan a mediciones no repetibles; dependientes del recinto y que no describen a la fuente. Una alternativa consiste en realizar la medición en un recinto anecoico, pero dada la escasa disponibilidad y alto costo de los mismos se decidió buscar otra solución.

En 1975, Moeller [9] desarrolló un método pulsado que no requiere de una cámara anecoica y que fácilmente cancela las reflexiones no deseadas. El transductor es excitado con una señal armónica limitada en el tiempo, de forma tal de contener varios ciclos (pulso sinusoidal). Al aplicar este pulso al transductor, la señal que llega al micrófono inicialmente cuenta con un transitorio, luego se establece un régimen pseudo-estacionario y finalmente aparecen las reflexiones. Durante el régimen pseudo-estacionario la senoide es igual a la que se registraría en una cámara anecoica o al aire libre utilizando una señal continua. Aplicando una ventana es posible extraer esta parte del registro y así poder 


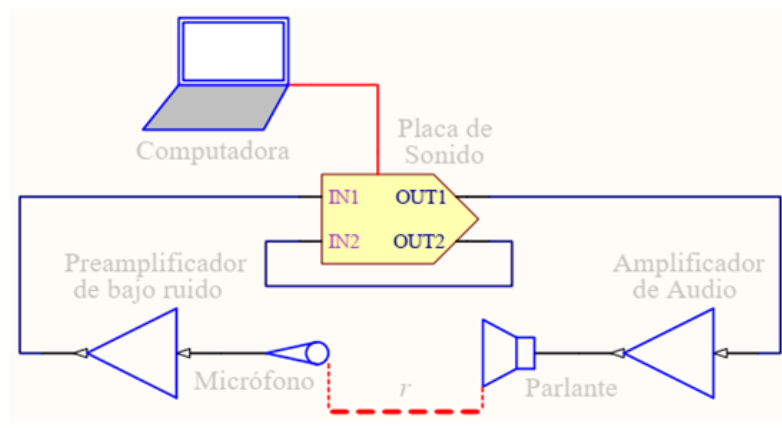

Fig. 6. Esquema del sistema de generación de tonos y captura de los pulsos acústicos

medir solamente el sonido directo. En el artículo original [9], el procedimiento se realizaba con un generador de señal, un osciloscopio, un sistema de ventana (time gating) y un detector de pico, todos independientes entre sí. La solución adoptada en este caso se resumió en una computadora para realizar el procesamiento (ventaneo, filtrado y detección de pico) de datos, una placa de sonido M-Audio Audiophile 2496 para generar los pulsos y recibirlos a través de un micrófono (ver Fig. 6).

Para la medición de respuesta en frecuencia, el sistema genera pulsos de $4 \mathrm{~ms}$ de duración por ambas salidas (OUT1 y OUT2) espaciados el tiempo necesario para permitir que las reflexiones se extingan. La salida OUT2 está directamente conectada a la entrada IN2 y sirve para dar la señal de disparo para la toma de datos. Esta toma se activa cuando IN2 detecta una señal creciente y superior a un umbral. El transductor reproduce cada pulso que luego es captado por el micrófono y digitalizado a través de la entrada IN1. Cada muestra obtenida es filtrada alrededor de la frecuencia bajo prueba y luego se aplica la ventana para extraer la señal pseudo-estacionaria. El procedimiento se repite para tener varias secuencias a ser promediadas y mejorar así la relación señal a ruido.

Para el relevamiento del patrón direccional, ubicamos al transductor en un trípode al cual adosamos un goniómetro. El micrófono no se mueve durante todo el ensayo y para asegurarse que quede centrado respecto al transductor se utiliza un puntero láser. Para ilustrar gráficamente el sistema implementado se muestran alguno de los registros obtenidos (Figs. 7-9). La primera de ellas corresponde al micrófono ubicado a cero grados respecto de la normal al transductor. El sonido directo es de amplitud más grande que el reflejado. La Fig. 8 es un detalle en el que se aprecia los transitorios de arranque y parada, así como la señal pseudo-estacionaria. El valor cuadrático medio de ésta es considerado como el representativo de la medición. Finalmente, en la Fig. 9 se observa el registro correspondiente a cuando el micrófono se ancuentra a 80 grados respecto a la normal. El sonido directo tiene ahora una magnitud mucho más pequeña, comparable al reflejado.

Aplicando el método descrito caracterizamos el prototipo cilíndrico. En la Fig. 10 mostramos los resultados para una frecuencia de $10 \mathrm{kHz}$ con el micrófono ubicado a $20 \mathrm{~cm}$ del eje del transductor y equidistante de los extremos. Es importante destacar que la máxima variación de presión

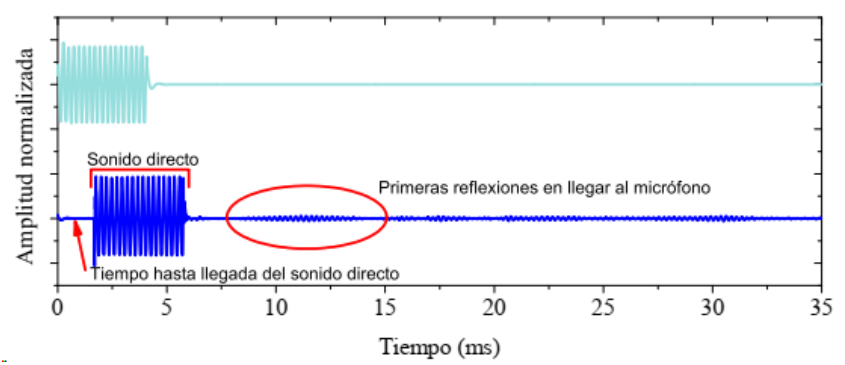

Fig. 7. Registros obtenidos en el prototipo plano.Traza superior: pulso emitido (IN2), traza inferior: señal registrada (IN1) a cero grados. La señal directa (de gran amplitud) arriba antes que los reflejos

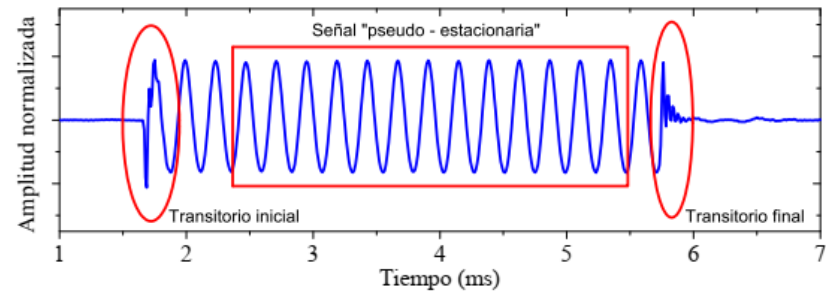

Fig. 8. Detalle de la señal recibida correspondiente a la Fig. 7 . Los transitorios de inicio y finalización son descartados. El valor cuadrático medio de la zona central es considerado como representativo de la señal directa

sonora respecto del valor medio es de $0,7 \mathrm{~dB}$. Por lo tanto, a lo largo de toda la circunferencia el sonido generado no tiene variaciones apreciables de nivel, aún a una frecuencia relativamente elevada $(10 \mathrm{kHz})$. Los radiadores convencionales suelen presentar lóbulos secundarios importantes en este rango.

Posteriormente medimos, a diferentes frecuencias, la presión sonora en función de la distancia $r$ al transductor y de la coordenada $z$, medida respecto del plano medio del mismo. Comparamos los resultados con las predicciones teóricas dadas por Williams [10]. La asignación de un radio en el modelo numérico es complejo debido a que en nuestro caso el radio de la frontera no está claramente definido. En efecto, dados los mecanismos de generación de sonido, los mismos se encuentran espacialmente distribuidos, no pudiéndosele asignar una ubicación unívoca. La mejor concordancia con los datos experimentales la encontramos asignando un radio de $21 \mathrm{~mm}$. La Fig. 11 muestra la simulación, por el método de elementos finitos, evaluada a $10 \mathrm{kHz}$ donde se indican los ejes a lo largo de los

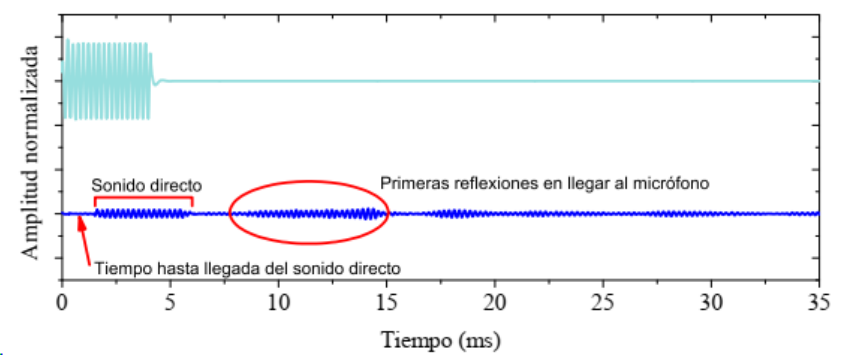

Fig. 9. Registros obtenidos en el prototipo plano. Traza superior:señal (IN2) emitida, traza inferior: señal recibida (IN1) a 80 grados. Nótese que la amplitud de la señal directa y la reflejada son del mismo orden. 


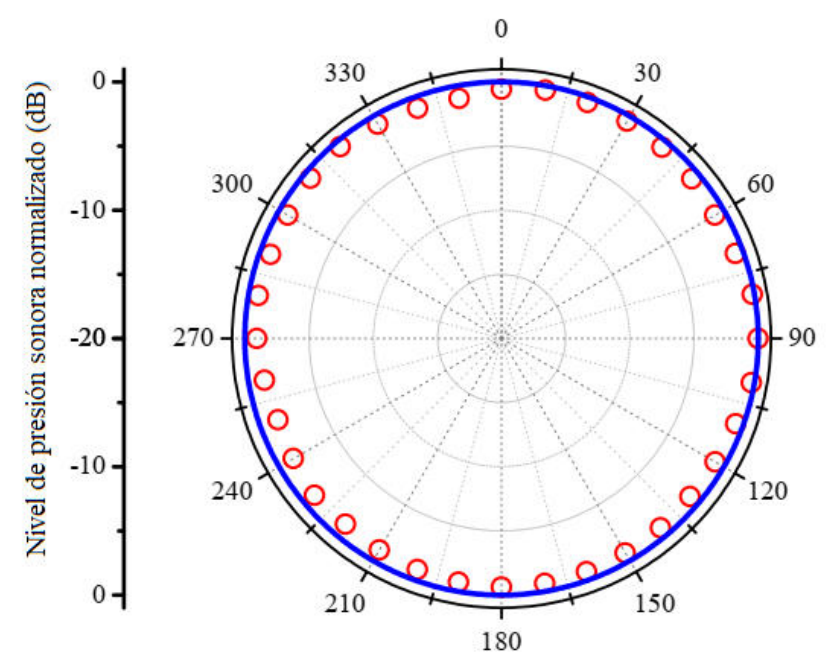

Fig. 10. Diagrama de emisión del transductor cilíndrico. Medida realizada a mitad de altura del mismo y a $20 \mathrm{~cm}$ de distancia

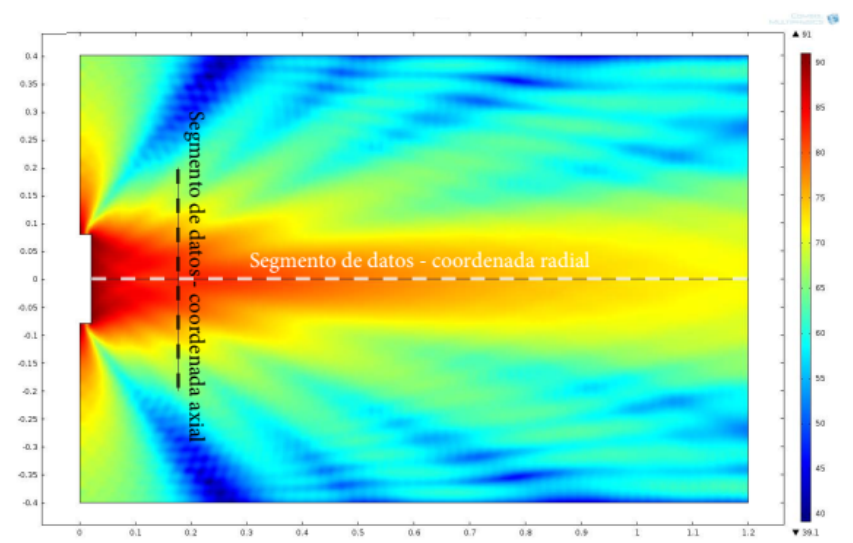

Fig. 11. Simulación del diagrama de emisión de un transductor cilíndrico finito a $10 \mathrm{kHz}$

cuales se relevó la presión acústica. En la Fig. 12 mostramos los resultados correspondientes a desplazamientos axiales. La simulación indica que la presión es aproximadamente constante en una zona central de extensión aproximadamente igual a la mitad del largo del transductor. Los resultados experimentales muestran una asimetría entre el extremo superior y el inferior. Esto probablemente se deba a que la forma de los cabezales del transductor no es idéntica para ambos extremos por razones constructivas. La asimetría, si bien notoria, no excede $2 \mathrm{~dB}$

La presión sonora asociada a una fuente cilíndrica de longitud infinita debe variar como $r^{-1 / 2}$. Como nuestra fuente es de largo finito, es dable esperar que a grandes distancias la presión varíe como $r^{-1}$ puesto que comienza a parecer una fuente esférica. La Fig. 13 muestra los resultados experimentales. Puede apreciarse que hasta unos $25 \mathrm{~cm}$ de la fuente, la misma se comporta como cilíndrica y "muy larga".

\section{APLiCACIÓN A CRISTAL SÓNiCO}

El transductor cilíndrico fue empleado por el Laboratorio de Acústica y Percepción Sonora (LAPSo, Universidad Nacional de Quilmes, CONICET) para caracterizar cristales sónicos [11], [12]. Los mismos constan de un arreglo

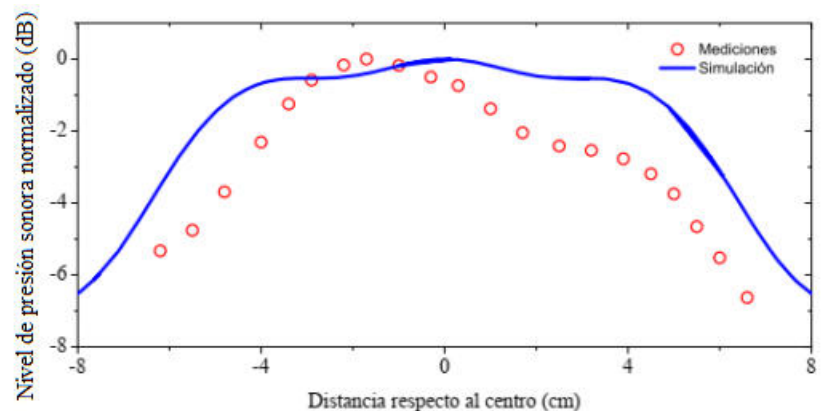

Fig. 12. Presión acústica en función de la posición vertical del micrófono. Trazo lleno: curva tomada de la Fig. 11, círculos: medidas experimentales

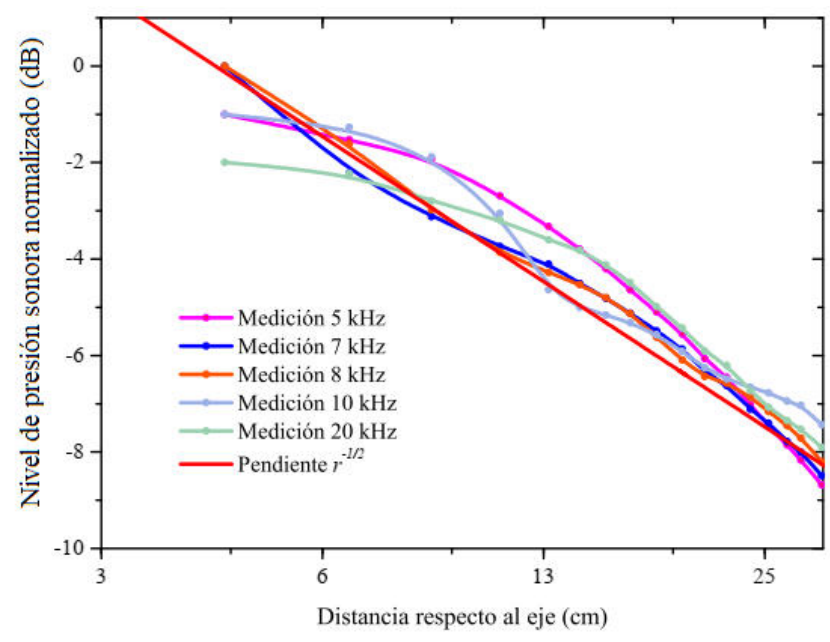

Fig. 13. presión acústica en función de la distancia al eje del transductor para varias frecuencias

periódico de tubos de aluminio. El análisis acústico de esta estructura muestra la existencia de rangos de frecuencias (bandas prohibidas) para los cuales la onda acústica queda confinada a la cavidad central, sin propagación efectiva en la estructura periódica. Un conjunto de tubos de aluminio de $25 \mathrm{~mm}$ de diámetro y $110 \mathrm{~mm}$ de altura son ubicados en los vértices de una matriz rectangular, separados sus centros 33 $\mathrm{mm}$. La matriz consta de 20 filas y 24 columnas. La región central, cuyas dimensiones son 10 filas y 14 columnas, carece de tubos. Esta zona actúa como cavidad resonante y en el centro de ella se ubica el transductor cilíndrico. El análisis teórico se realizó con una fuente cilíndrica dado que el desarrollo resulta más simple. El estudio predice la aparición de bandas de frecuencias permitidas (el sonido es radiado fuera de la cavidad) y prohibidas (la cavidad actúa como si fuera "cerrada"). La respuesta en frecuencia obtenida con un micrófono en la cavidad mostró un incremento de presión acústico en el rango de frecuencias correspondiente a la banda prohibida, mostrando que la cavidad "resuena" [11], [12]. El uso del transductor iónico presentado y estudiado en este trabajo permitió verificar el comportamiento acústico del cristal sónico excitado con un frente de onda cilíndrico.

\section{CONCLUSIONES}

Los resultados experimentales muestran que, dado que el campo de velocidades del aire en las vecindades de 
un transductor iónico apunta en la dirección normal a los electrodos, es posible obtener un frente de onda que siga la forma de los mismos. Esto brinda una atractiva flexibilidad para tener fuentes sonoras con un diagrama de irradiación de forma compleja, engorroso de conseguir por métodos convencionales. Estas aplicaciones son específicas para experiencias de laboratorio realizadas a corta distancia del emisor puesto que en el campo lejano las diferencias entre dos distintos tipos de emisores tienden a diluirse.

\section{AGRADECIMIENTOS}

Este trabajo fue apoyado por los subsidios de la Universidad de Buenos Aires (UBACyT 20020160100052BA) y de la ANPCyT (PICT 2016-2204).

\section{REFERENCIAS}

[1] L. Beranek and T. Mellow, "Acoustics: sound fields and transducers," Academic Press, 2012.

[2] F. Bastien, "Acoustics and gas discharges: applications to loudspeakers," Journal of Physics D: Applied Physics, vol. 20, no. 12, p. 1547 1987.

[3] P. Bequin, K. Castor, P. Herzog, and V. Montembault, "Modeling plasma loudspeakers," The Journal of the Acoustical Society of America, vol. 121, no. 4, pp. 1960-1970, 2007.

[4] Y. Sutton, P. Johnson, G. Naidis, D. Sharp, and N. Braithwaite, "A numerical and experimental investigation of an axially symmetric rf plasma," Journal of Physics D: Applied Physics, vol. 45, p. 455201, 2012.

[5] M. Robinson, "A history of the electric wind," Am. J. of Phys., vol. 30, no. 5, pp. 366-372, 1962.

[6] D. M. Tombs, "Corona wind loud-speaker," Nature, vol. 30, p. 923, 1955.

[7] K. Matsuzawa, "Sound sources with corona discharges," The Journal of the Acoustical Society of America, vol. 54, no. 2, pp. 494-498, 1973.

[8] P. Gómez, M. D’Onofrio, and G. Santiago, “Acoustic characteristics and distortion of an ionic loudspeaker," IEEE Latin America Transactions, vol. 13, pp. 578-582, 2015.

[9] H. Moller and C. Thomsen, "Electroacustic free field measurements in ordinary rooms using gating techniques," Audio Engineering Society Convention, vol. 52, 1975

[10] W. Williams, N. G. Parke, D. A. Moran, and C. H. Sherman, "Acoustic radiation from a finite cylinder," The Journal of The Acoustical Society of America, vol. 36, no. 12, pp. 2316-2322, 1964.

[11] A. Alberti, P. M. Gómez, I. Spiousas, and M. C. Eguía, "Broadband resonant cavity inside a two-dimensional sonic crystal," Applied Acoustics, vol. 105, pp. 1-5, 2016.

[12] A. Alberti, I. Spiousas, P. M. Gómez, and M. C. Eguía, "Resonance switching in a large sonic crystal cavity," Applied Acoustics, vol. 116, pp. 390-393, 2017. 